\title{
Management of abusive and unfair Internet access by quota-based priority control is
}

\author{
Tsung-Ching Lin a , Yeali S. Sun ${ }^{\mathrm{b}}$, Shi-Chung Chang ${ }^{\mathrm{a}, *}$, \\ Shao-I Chu ${ }^{\text {a }}$, Yi-Ting Chou ${ }^{a}$, Mei-Wen $\mathrm{Li}^{\mathrm{c}}$ \\ ${ }^{a}$ Department of Electrical Engineering, National Taiwan University, Taipei, Taiwan \\ ${ }^{\mathrm{b}}$ Department of Information Management, National Taiwan University, Taipei, Taiwan \\ ${ }^{\mathrm{c}}$ Computer and Information Network Center, National Taiwan University, Taipei, Taiwan
}

\begin{abstract}
In a free of charge or flat-rate Internet access environment, there often exists abusive and unfair usage of network resources. In this paper, the Internet access by the dormitory users at the National Taiwan University (NTU) serves as a conveyer problem. A quota-based service architecture combined with priority scheduling is developed to resolve the problem of unfair and abusive Internet access encountered in the NTU dormitory networks. The goal is to meet the basic traffic demands of the majority users while limiting abusive usage from ignorant heavy users. Two classes of services are provided. The regular class sets a quota on each user's traffic volume per quota control period. Out-ofprofile or possibly abusive traffic is directed to a lower priority service class called the custody service and served when there is an excess bandwidth. The new policy and schemes are implemented on a quality of service router, a meter reading server, an accounting server and a Web-based service management server with minimal intrusion of user privacy and least disturbance to the existing service offering. The experimental results show that the original congestion at the bottleneck link was alleviated with a $48.9 \%$ reduction of the average packet drop rate. Abusive Internet access by the top $2 \%$ heavy users is greatly reduced by $57.82 \%$. Astonishingly, over $91 \%$ users are pleased with this new policy and their network usages all increase. This is a win-win result. Under the new policy and implementation, users paying the same amount of service fee are now able to fairly share resources. A mathematical model for prediction of control effects is also presented. The experimental results are consistent with the prediction of the model.
\end{abstract}

(c) 2003 Elsevier B.V. All rights reserved.

Keywords: Priority; Quota; Internet access control; Abuse reduction; Fairness; Experiment on production network

\footnotetext{
This work was supported in part by the National Science Council of the Republic of China under Grants NSC88-2215-E002-026, NSC89-2215-E-002-037 and NSC-90-2213-E-002-078.

${ }^{*}$ Corresponding author. Tel.: +886-2-23635251x245; fax: 886-2-23638247.

E-mail address: scchang@cc.ee.ntu.edu.tw (S.-C. Chang).
}

\section{Introduction}

In a free of charge or flat-rate Internet access environment, there often exists abusive and unfair usage of network resources. In this paper, we report a real case of abusive usage by a small group of users in National Taiwan University's (NTU's) dormitory networks, and the success of using a simple yet effective quota-based priority control 
scheme to resolve the problem. In the case of the NTU dormitory networks, most of such abusive usage carries unimportant, excessive and even illegal data. Abusive use of limited resources often leads to poor network performance, e.g., high packet loss rate and long delay. It is obviously unfair to the other users suffering the consequence. To achieve fairness between users who share the same network resources, possibly paying the same amount of service fee, the best tactic is to prevent selfish users from abusing the resources.

In the past, many researchers have studied the fairness problem of the resource sharing in packet switched networks. Different notions of fairness were proposed. The most commonly used notion of fairness [1] is the max-min fairness. It aims at allocating as much as possible to small users. However, it has been pointed out by [2] that such fairness may be at the expense of some network efficiency because it emphasizes maintaining high values for the smallest rates. In [3], the authors advocated the notion of proportional fairness to maximize the overall utility of rate allocations assuming that Internet traffic is elastic and the utility of each traffic flow is logarithmic. In [4], the potential-delay minimization fairness was proposed with the goal of finding the flow allocations to minimize the total delay. In [5], the logarithmic proportional fairness is defined to attain high network utility and small total delay. All these different methods focus on the rate control in a short time period, e.g., a few seconds or less, to achieve the fairness they defined. In our case, we seek for a fair usage control over a larger time scale such as a day or longer.

There are very few studies on user behavior and demands in the literature based on the actual data from production networks. In the Internet Demand EXperiment or INDEX project [6], they conducted a market and technology trial for the ISDN lines with only 70 subjects or participants at the University of California at Berkeley. In the experiments, differentiated-quality services were offered with different prices to reflect the resource cost. They showed that user demands for Internet access are very sensitive to price as well as quality. There exists possibly a large and persistent difference in demand among users. In [7], they studied a large-scale user behavior on Internet access (over 60,000 users) through a detailed investigation of some content delivery systems including Web servers and peer-to-peer networks at the University of Washington. As pointed out in this study, in order to provide better Internet services to the general users, it is increasingly important to gain detailed understanding of user behavior from the production systems and networks.

In response to the poor Internet access performance experienced by the majority of dormitory users, the university campus network administration initiated the Virtual Internet Pricing (VIP) project to resolve the problem of unfair and abusive use of the network resources in the dormitory networks. As a production network, minimal intrusion of user privacy and least disturbance to the existing service offering are two of the most important factors in addition to costs that the network administration considers when selecting the solutions and changes to the existing networks. In addition, we think it is equally important that the adopted scheme or policy must be simple enough for average users to understand. In order to plan and manage the new policy/scheme effectively, it is essential that we have an in-depth understanding of user behavior as well as a thorough grasp of the details of the existing and the state-of-the-art technology. After careful examination, a quota based control scheme combined with priority scheduling at the bottleneck link is adopted.

Quota based control has been a successful scheme to achieve fairness by limiting individual user's usage of resources within a period of time in many applications, for example $[8,9]$. In the case of controlling network usage in a flat-rate or free-ofcharge network, a fixed amount of quota in traffic volume is set for every user effective within the quota control period. The in-profile traffic is served with a higher priority than the out-profile traffic. When a user uses up his/her quota, the subsequent traffic from the user is directed to a lower priority class of service when entering the controlled bottleneck link until the end of this control period. The quota control scheme sets a hard limit on each user's access of the network. It 
effectively prevents users from abusing network resources and is generally considered fair.

Priority scheduling is a simple method in allocating resources among contending demands. In the IETF DiffServ architecture [10], a prioritybased service called Assured Forwarding (AF) Per-Hop-Behavior (PHB) was defined. In AF, there are four service classes in which services with higher priority always have better quality of service than services with lower priority. In [11], they proposed a method to transparently assign different priority classes with different tariffs to users in a local area network environment based on the user demands. However, in a free or flat rate charging environment, how to assign different service classes to different user groups is critical to achieve a fair resource allocation.

By quota limitation, we are able to regulate heavy users' sending excess traffic to congest the network, which severely curtails the use by regular users. By priority scheduling, all users possibly paying the same service fee are protected with basic, minimum guarantees of service. In the meantime, the heavy users are allowed to transmit more than allowed traffic marked as lower priority to make use of the excess network resources. Thus, the scheme simultaneously achieves fairness and efficiency in resource sharing and utilization. In addition, the scheme is easy to understand and has the least disturbance to the existing network installation and service offering.

Under the scheme, several important issues have arisen. First, how to determine the amount of quota given different levels of user demands? Second, how this affects the fairness, overall network performance and the quality of service received by the users. In this paper, a mathematical model is proposed for the analysis and design of the quotabased priority control scheme for Internet access. Both numerical and empirical results are presented to show the significant fairness improvement by eliminating resource abuse.

Our quota-based priority control applies to Internet accesses from LAN environments where the accesses by individual users are free of charge or charged at a flat rate. Examples of free-ofcharge Internet access include campus, government and corporate LANs, where the organization pays for individual users' Internet access as a whole, while keeping no record of individual usage. LANs of household, community or small business users are very often charged at a flat rate. In these environments, when the bandwidth for Internet access is not too much larger than the total user demands, the few abusive users may lead to poor performance in Internet access and unfairness for the majority users. Our quota-based priority control scheme readily serves as a simple but effective remedy to the abusive usage in these realistic network environments.

The remaining of this paper is organized as follows. Section 2 describes the issues of unfair and abusive Internet access by users of the NTU dormitory networks. In Section 3, we propose a mathematical model for the analysis and design of the quota-based priority control scheme. System implementations are described in Section 4. Section 5 presents the experimental results to validate the design in Section 3. Finally, we give some concluding remarks about the Internet access control scheme.

\section{Unfair and abusive Internet access}

The campus network service and Internet access at NTU are largely government subsidized. Students living in the dormitories paid a flat charge of less than US $\$ 9$ per semester for such services. Fig. 1 depicts the configuration of the dormitory networks and the link to the NTU campus network and the Internet before our quota-based priority control was installed, referred to as the "baseline" configuration. There are 5355 student users residing in 14 dormitories in two separated areas. Among them, $76.6 \%$ are undergraduates and $23.4 \%$ are graduate students; and there are $14.1 \%$ and $85.9 \%$ female and male students, respectively. Each user is provided with an Unshielded Twist Pair (UTP) Ethernet 10/100 Mbps access point and is assigned an Internet IP address. Local area networks of individual dormitories, i.e., dormitory networks, consist of some 100 Mbps Ethernet switches per floor of each dormitory and per dormitory. All the other local area networks in NTU campus, which are mainly for administrative 


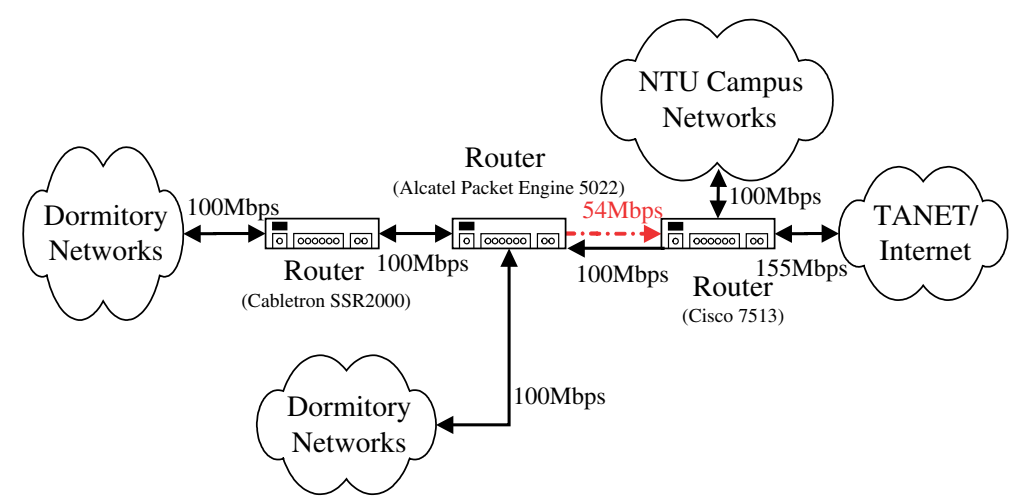

Fig. 1. The original Internet access architecture for the dormitory users.

and academic purposes, are referred to as NTU campus networks thereafter. Dormitory network traffic connects to the NTU campus network and the Taiwan Academic Network (TANet)/Internet by an Alcatel Packet Engine 5022 router and a Cisco 7513 router. The link between the two routers is a $100 \mathrm{Mbps}$ fast Ethernet link. In addition to the unfairness and congestion problems, the administrators of the university campus network also face the problem that the total traffic emitted from the dormitory networks generates more than $50 \%$ of the ATM 155 Mbps campus backbone. Initially, they decided to impose rate limit to the traffic from this cluster of dormitories. A maximum of $54 \mathrm{Mbps}$ was set to limit the outbound traffic from the dormitory networks.

There are two levels of management over the dormitory networks: dormitory and campus. At the dormitory level, each dormitory has a student manager, who resides in the dormitory and manages its LAN. They play the roles of IP address managers, network technology consultants and delegates of dormitory students for campus network management. At the campus level, the division of network management of the computer and information network center is responsible for the overall campus network management of campus, including all dormitories. There are regular coordination meetings between dormitory administrators and campus network managers. Through such meetings, new network management policies are announced to the student managers, and users' comments are fed back to the campus network managers.

Netflow $^{\mathrm{TM}}$ [12], a traffic monitoring and collection mechanism of the Cisco 7513 router, monitors the traffic through it. Every 10 minutes, Netflow summarizes traffic data of individual IP flows. Each IP flow is identified by its source and destination IP addresses, source and destination port numbers and the protocol type (TCP or UDP). Based on the collected Netflow data, an accounting server computes various traffic flow statistics such as the per-IP traffic volume, total traffic toand-from TANet access, traffic between dormitory and campus networks, traffic of individual dorms, traffic by application type, and packet drop rates.

\subsection{Traffic characteristics}

Measurements of traffic flows in and out of the dormitory networks show that the outbound traffic is constantly congested under the $54 \mathrm{Mbps}$ bandwidth allocation while the inbound traffic is on average at $17 \%$ utilization of the $100 \mathrm{Mbps}$ bandwidth. The 54 Mbps link is considered congested when the packet drop rate of a 10-minute time slot is more than 5.4 Mbps. Shown in Fig. 2 are the representative daily outbound throughput and drop rate at the Cisco 7513 router over a typical and uneventful week. It can be seen in Fig. 2 that the congestion lasts over 17 hours, from 11 a.m. to 4 a.m. the next day. The average daily network utilization is $99.57 \%$ with an average 


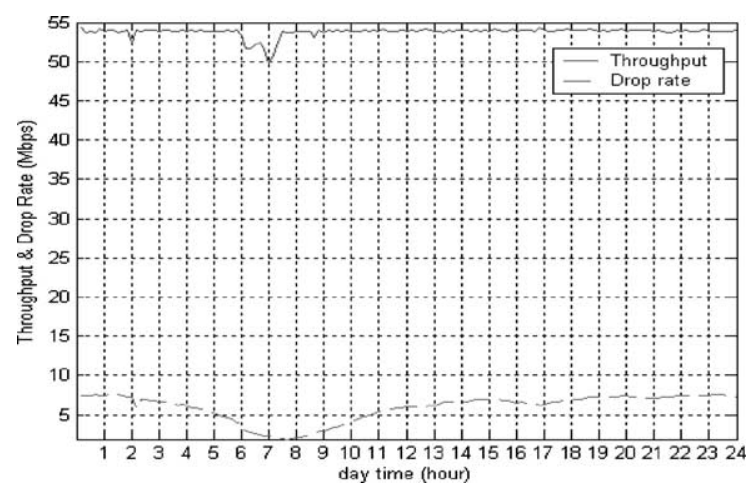

Fig. 2. Drop rate and throughput of outbound traffic.

packet drop rate of 5.94 Mbps. Such network service quality is definitely unsatisfactory. The outbound traffic is obviously a focus of the dormitory network management.

The outbound traffic comprises the traffic from the dormitory networks to the Internet referred to as the "Internet access" traffic and the traffic from the dormitory networks to the NTU campus networks referred to as the "intranet access" traffic. Our analysis in Fig. 3 shows that the Internet access traffic has an average of $36.58 \mathrm{Mbps}$, which constitutes more than two thirds of the outbound traffic. The "intranet access" traffic has an average of $17.18 \mathrm{Mbps}$. The almost constant congestion is mainly caused by the heavy Internet access.

Further analysis by application shows that $99.88 \%$ of the Internet access traffic is the TCP traffic. The specific application of a TCP packet is

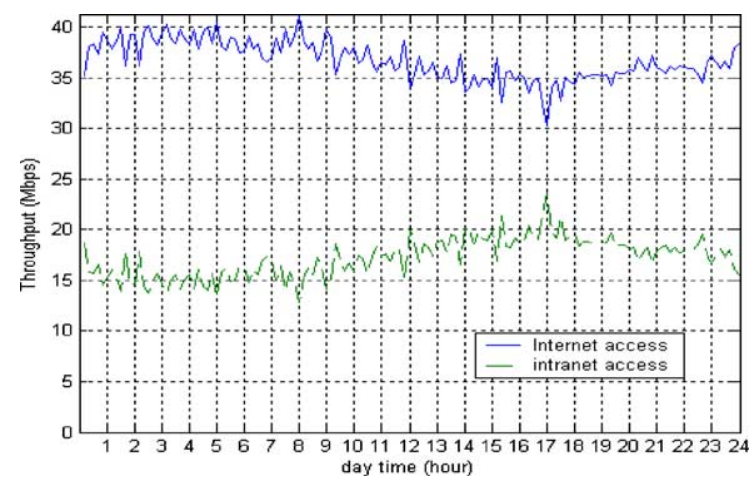

Fig. 3. Internet and intranet traffic components in outbound traffic.
Table 1

Traffic analysis by application

\begin{tabular}{lll}
\hline Application & Campus & \\
\cline { 2 - 3 } & NTU & $\begin{array}{l}\text { UC Berkeley } \\
\text { (INDEX project) }\end{array}$ \\
\hline OTHERS & 95.7 & 32.5 \\
HTTP & 3.2 & 43 \\
HTTPS & 0.62 & 2 \\
Telnet/SSH & 0.22 & 4 \\
FTP & 0.10 & 11 \\
SMTP & 0.084 & 0.5 \\
POP3 & 0.057 & 3 \\
NNTP & 0.0022 & 2 \\
X-Windows & 0 & 2 \\
\hline
\end{tabular}

identified by checking the port number through which the packet is transmitted. Table 1 lists the eight most popular applications in the NTU dormitory network and compares to those in the INDEX project of the University of California, Berkeley [6]. Note that $95 \%$ of NTU traffic bears not-well-known port numbers, whose application is classified as "OTHERS." The "OTHERS" traffic in UC Berkeley's campus network traffic is only $32.5 \%$. A high percentage of the "OTHERS" traffic is definitely undesirable for the campus network manager because it may hide some usage that intentionally avoids being easily managed.

\subsection{User characteristics}

A representative distribution of the daily Internet access traffic among users is presented in Fig. 4. It clearly shows a three-peak profile. We thus define three user groups accordingly: (1) light users (LUs), each contributing less than 3162 bytes per day, (2) normal users (NUs), each having daily usage between 3162 bytes and 100 megabytes, and (3) heavy users (HUs), each using more than 100 megabytes per day. Note that the HU group contributes $96.7 \%$ of the Internet access traffic and should be a target for management. We further divide the HU group into three subgroups: 2 GB-heavy users (2GHUs), 1 GB-heavy users (1GHUs), and 100 MB-heavy users (100MHUs) with daily usage of over $2 \mathrm{~GB}$, between 1 and $2 \mathrm{~GB}$, and between $100 \mathrm{MB}$ and $1 \mathrm{~GB}$, respectively. 


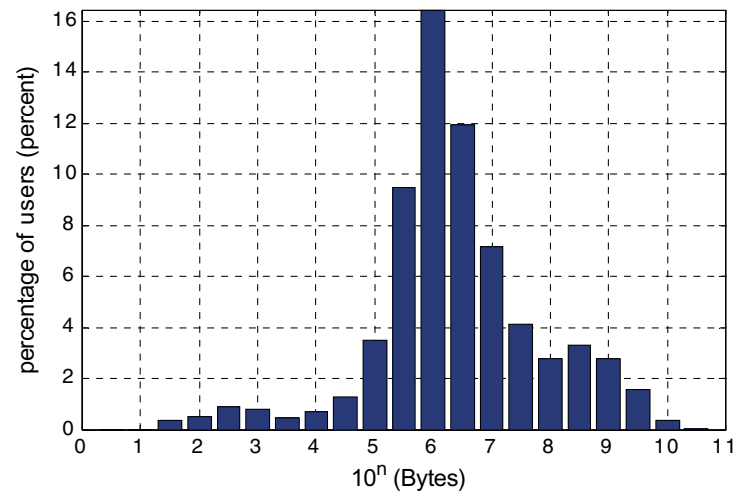

Fig. 4. A representative daily volume of Internet access distribution.

The average daily usage for Internet access over all users is around $81.7 \mathrm{MB}$ with a large standard deviation of $471.83 \mathrm{MB}$. As shown in Table 2 and Fig. 4, NUs and LUs account for $91.8 \%$ of the user population and create only $4.3 \%$ of the daily traffic volume. Fig. 5 gives typical daily throughput profiles of the five user groups. The usage behavior of NUs is reasonable and normal while peak hours were during 9 p.m. to 3 a.m., the most convenient hours for student users to access the Internet.

In contrast to LU and NU groups, all empirical data indicates that the Internet access link was abused by the HU group, which leads to congestion. Table 2 shows that $2 \mathrm{GHU}$ and $1 \mathrm{GHU}$ groups account for only $2 \%$ of the user population but create $66.2 \%$ of the total traffic load. Statistics of individual IPs indicate that most of the HUs

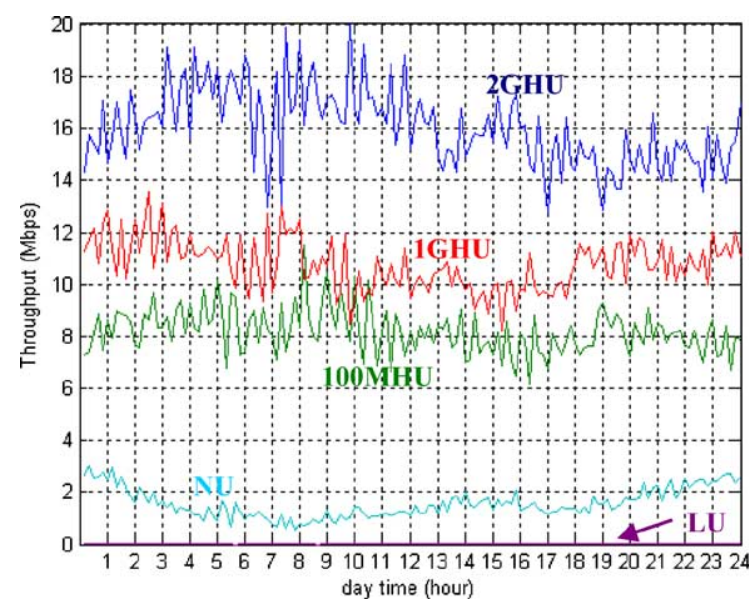

Fig. 5. Throughput profiles of different user groups.

continuously send outbound Internet traffic around the clock. The 2GHUs and 1GHUs contribute heavy traffic flows all day, and it is believed that these heavy flows are due to some unregistered Web or FTP sites owned by 2GHUs and 1GHUs. As a result, LUs and NUs have also to suffer from congestion and high packet losses, especially during the peak hours, which is highly unfair to them. The campus network management would like to regulate such abnormal Internet access behavior.

\subsection{Challenges to network management}

User behavior of unfair and abusive Internet access at the NTU dormitory networks has been

Table 2

The usage statistics among given groups

\begin{tabular}{llll}
\hline User group & Statistics & & \\
\cline { 2 - 4 } & Users number & Total volume (bytes) & Mean (bytes) \\
\hline $2 \mathrm{GHU}$ & $5355(100 \%)$ & $395,100,888,447(100 \%)$ & $3,846,103,572$ \\
\multirow{2}{*}{$1 \mathrm{GHU}$} & 45 & $173,074,660,758$ & $1,429,553,853$ \\
& $(0.84 \%)$ & $(43.81 \%)$ & $353,783,149$ \\
$100 \mathrm{MHU}$ & 62 & $88,632,338,859$ & $5,586,235$ \\
\multirow{2}{*}{$\mathrm{NU}$} & $(1.16 \%)$ & $(22.43 \%)$ & 170 \\
& 329 & $116,394,655,881$ & \\
\hline
\end{tabular}


analyzed in Sections 2.1 and 2.2. The abusive usage of limited resources by a small number of users leads to poor network performance, and the majority of the users have to suffer from the consequences. Such abusive and unfair phenomena are common in networks that offer a free or flat rate charge. In a network of many IP users, 5335 in NTU dormitory networks, the true demands of individual users are hardly known to the network manager. On the one hand, a network manager would like to encourage normal usage of network services; On the other hand, a network manager would like to prevent the network from being congested. Without knowing the true demands, homogeneous and strict control of usage among individual users may lead to fake fairness or inefficient bandwidth utilization.

In any solution to abuse reduction and fairness improvement, minimum intrusion of user privacy and least disturbance to the existing service are two of the key criteria of network management. Cost is always a constraint in improving network service. It is almost a must to consider control schemes simple for users to understand, and to have least disturbance to the existing network management practice. Challenges in our attempt to improve fairness, reduce abuse and identify user behavior are summarized as follows:

1. How to define fairness and abuse without knowing the true demands of individual users?

2. How to identify the sources of abusive usage with the least intrusion of privacy?

3. How to eliminate abusive usage without discouraging normal usage?

4. How to improve the fairness among different users through a soft and homogeneous measure?

5. How to alleviate congestion by fairness improvement and abuse reduction?

6. How to exploit the hardware and software of the legacy production network and off-theshelf hardware and software for economic and easy implementation and maintenance of the solution?

7. How to keep the Internet service reliable and continuous while a new control system is implemented?
8. How to design the models to realize the control effects: fairness improvement, abuse reduction and understanding of user behavior?

9. How to analyze the relations among control variable, dependent variables and control effects?

10. How to discover, in depth, users' behavior without the intrusion into the application-usage of individual users?

\section{Priority control system design}

To effectively meet the challenges of improving the unfair and abusive Internet access and understanding user behavior with least disturbance, we shall design in this section a simple control scheme that combines the ideas of quota limitation and the priority differentiation. Our general goal is to maximize total user utility [14] of Internet access under the constraint of limited network resources. Our philosophy is to assure every user's basic service and to regulate heavy users' abusive usage by a homogeneous quota allocation; through a prioritized service, a user can still access the Internet when out of quota but at a lower service priority.

Our prioritized service consists of two service classes: regular and custody. The regular service has a higher priority than the custody service for data transmission. There is a volume quota for each user's regular service, which is designed to meet majority users' essential demands while limiting abusive usage of quality service. The custody class has no quota limit, which allows heavy users to access the Internet at a lower quality. The default class of service is regular. An ideal design and implementation would be that the majority users are unaware of the introduction of the control scheme but enjoy a much improved service quality. Under a given Internet access bandwidth, the design problem boils down to evaluating how the quota per IP user may affect network performance and service quality and hence to the determination of quota value. Exploiting the empirical data of user characteristics, network performance and architecture collected from the baseline network, where no priority is installed, we now construct a 
mathematical model of the relationship among the quota, user traffic demands and the network performance. The model facilitates the quota design and the analysis of how the priority control scheme may improve unfair and abusive Internet access.

\subsection{Individual user model}

Let the volumes of the Internet and intranet traffic transmitted by user $n$ at time slot $l$ in the baseline network be denoted as $v_{B I \_n l}$ and $v_{B i \_n l}$, $n \in\{1,2, \ldots, N\}$ and $l \in\{1,2, \ldots, L\}$, where $L$ is the total number of time slots in a day, $N$ is the total user population, $I$ means the Internet access, $i$ means the intranet access and $B$ means the baseline network. The drop ratio at time slot $l$ is denoted as $d_{B_{-} l}, l \in\{1,2, \ldots, L\}$. A user's original transmission demand includes both transmitted and dropped packets. Traffic arrival volumes from user $n$ for Internet and intranet accesses at time slot $l$ are estimated as

$$
\begin{aligned}
& \hat{a}_{B I \_n l}=v_{B I \_n l}\left(1+d_{B_{-} l}\right), \\
& \quad l \in\{1,2, \ldots, L\}, n \in\{1,2, \ldots, N\},
\end{aligned}
$$

and

$$
\begin{aligned}
& \hat{a}_{B i \_n l}=v_{B i \_n l}\left(1+d_{B \_l}\right), \\
& \quad l \in\{1,2, \ldots, L\}, n \in\{1,2, \ldots, N\} .
\end{aligned}
$$

Let the volume quota be $H$. If the cumulative regular Internet traffic volume of a user exceeds the quota $H$, the subsequent Internet access of the user will be downgraded to the custody service only. For regular Internet access, the traffic arrival volume from user $n$ at time slot $l$ is estimated as

$$
\begin{gathered}
\hat{a}_{H I \_n l}= \begin{cases}\hat{a}_{B I \_n l} & \text { if } \sum_{j=1}^{l-1} \hat{v}_{H I \_n j}<H, \\
0 & \text { otherwise, }\end{cases} \\
l \in\{1,2, \ldots, L\}, n \in\{1,2, \ldots, N\},
\end{gathered}
$$

where $\hat{v}_{H I \_n j} n \in\{1,2, \ldots, N\}$ and $j \in\{1,2, \ldots, L\}$, is the regular-class Internet traffic volume successfully transmitted by user $n$ at time slot $j$.

Thus, the total regular traffic arrival volume from user $n$ at time slot $l$ is

$$
\begin{aligned}
& \hat{a}_{H \_n l}=\hat{a}_{H I \_n l}+\hat{a}_{B i \_n l}, \\
& \quad l \in\{1,2, \ldots, L\}, n \in\{1,2, \ldots, N\},
\end{aligned}
$$

and the total regular-class traffic arrival volume from all users at time slot $l$ is calculated as

$\hat{A}_{H_{-} l}=\sum_{n=1}^{N} \hat{a}_{H_{-} n l}, \quad l \in\{1,2, \ldots, L\}$,

and the estimate of the traffic arrival rate at time slot $l$ is calculated as

$\widehat{R}_{H_{-} l}=\frac{\hat{A}_{H_{-} l}}{T}, \quad l \in\{1,2, \ldots, L\}$,

where $T$ is the length of each time slot. Once the estimated traffic arrival rate (i.e., the offered load) exceeds the allocated link bandwidth $B$, i.e., $\widehat{R}_{H_{-} l} \geqslant B$, we consider that congestion occurs. The drop ratio of the outbound traffic is predicted as

$$
\begin{aligned}
\hat{d}_{H_{-} l} & = \begin{cases}\left(\widehat{R}_{H_{-} l}-B\right) / B & \text { if } \widehat{R}_{H_{-} l}>B, \\
0 & \text { otherwise, }\end{cases} \\
l & \in\{1,2, \ldots, L\} .
\end{aligned}
$$

It is assumed that the regular Internet access and intranet access suffer from the same drop ratio. Hence, the regular traffic volumes successfully transmitted by user $n$ for Internet and intranet accesses at time slot $l$ are estimated as

$\hat{v}_{H I \_n l}= \begin{cases}\hat{a}_{H I \_n l}\left(1-\hat{d}_{H_{-} l}\right) & \text { if } \sum_{j=1}^{l-1} \hat{v}_{H I \_n j}<H, \\ 0 & \text { otherwise }\end{cases}$

and

$$
\begin{aligned}
& \hat{v}_{H i \_n l}=\hat{a}_{H i \_n l}\left(1-\hat{d}_{H_{-} l}\right), \\
& \quad l \in\{1,2, \ldots, L\}, \quad n \in\{1,2, \ldots, N\},
\end{aligned}
$$

and the estimate of the throughput at time slot $l$ is calculated as

$\hat{X}_{H_{-} l}=\frac{\sum_{n=1}^{N} \hat{v}_{H I_{-} n l}+\hat{v}_{H i \_n l}}{T}, \quad l \in\{1,2, \ldots, L\}$,

where $T$ is the length of each time slot. 
In summary, we have developed a model to predict, under a given quota $H$, every user's behavior and network performance within a time slot, including the drop ratio (Eq. (1)), per user's volume usage (Eq. (2)) and the throughput (Eq. (3)). Predictions of all time slots can therefore be obtained.

\subsection{Aggregate user model}

Based on Section 3.1, the means and the standard deviations of the regular traffic volumes transmitted by users for Internet and intranet access are calculated as

For Internet access

$\hat{\bar{v}}_{H I}=\frac{\sum_{n=1}^{N} \hat{v}_{H I \_n}}{N}, \quad \widehat{S}_{H I}=\sqrt{\frac{\sum_{n=1}^{N}\left(\hat{v}_{H I \_n}-\hat{\bar{v}}_{H I}\right)^{2}}{N-1}}$,

and

For intranet access

$\hat{\bar{v}}_{H i}=\frac{\sum_{n=1}^{N} \hat{v}_{H i \_n}}{N}, \quad \widehat{S}_{H i}=\sqrt{\frac{\sum_{n=1}^{N}\left(\hat{v}_{H i \_n}-\hat{\bar{v}}_{H i}\right)^{2}}{N-1}}$,

where $\hat{v}_{H I \_n}=\sum_{l=1}^{L} \hat{v}_{H I \_n l}$ and $\hat{v}_{H i \_n}=\sum_{l=1}^{L} \hat{v}_{H i \_n l}$, $n \in\{1,2, \ldots, N\}$.

To verify the fairness improvement and abuse reduction, we now formally define the abuse index as the total volume (number of bytes) transmitted by top $2 \%$ heavy users and the unfairness index as coefficient of variation of users' daily volume usage, which is calculated as the ratio of standard deviation and mean. These two indices are shown as:

Abuse index $=\sum_{n \in N_{98 \%}} \hat{v}_{H I\lrcorner n l}$,

Unfairness index $=\frac{\widehat{S}_{H I}}{\overline{\bar{v}}_{H I}}$,

where $N_{98 \%}$ is the set of users whose daily volume usage is in the top $2 \%$ of all users. As a result, we have the relationship between the quota and the aggregate user behavior, including mean of transmitted volume, standard deviation of transmitted volume, abuse index and unfairness index.
Table 3

Simulation results for Internet access

\begin{tabular}{lrrr}
\hline Information & \multicolumn{2}{l}{ Quota } & \\
\cline { 2 - 4 } & Infinite & \multicolumn{1}{c}{ 2 GB } & \multicolumn{1}{c}{ GB } \\
\hline Standard deviation (MB) & 449.03 & 282.66 & 186.49 \\
Mean volume (MB/user) & 73.78 & 68.93 & 52.97 \\
Unfairness index; & 6.09 & 4.10 & 3.52 \\
$\quad$ Coefficient of Variation & & & \\
Abuse index; Usage of top & 261.71 & 174.30 & 98.60 \\
$2 \%$ users (GB) & & & \\
\hline
\end{tabular}

\subsection{Prediction of control effects of quota}

We apply the user behavior and network performance models to predict the control effect of our proposed scheme. We construct a numerical experiment with the following parameters: the quota $H$ has two values $2 \mathrm{~GB}$ and $1 \mathrm{~GB}$, which satisfy at least $99 \%$ and $98 \%$ users in the baseline network respectively, the link bandwidth $B$ is 54 Mbps, and the total user population is 5355 . Interested readers may refer to the Appendix for the flow chart of the numerical experiment. Following the above models, we predict controlled user behavior listed in Table 3. Results show that a $42.2 \%$ improvement of unfairness and a $62.3 \%$ reduction of abusive usage as compared to the baseline network behavior.

In this section, we answered challenge (1) by formally defining abuse and unfairness indices. Our design of the quota-based priority control scheme addressed challenges (3)-(5) and the model constructed answered challenge (8). Our model predicts two expected achievements. The first is to satisfy every user's basic demand. The second is to allow the majority of non-heavy users to be unaware of the execution of combined quota and prioritized service but to enjoy the better network performance.

\section{Implementation over a production network}

To realize the control policy proposed in Section 3, we design an implementation system architecture as depicted in Fig. 6. For economic, reliable and easy implementation and maintenance, 


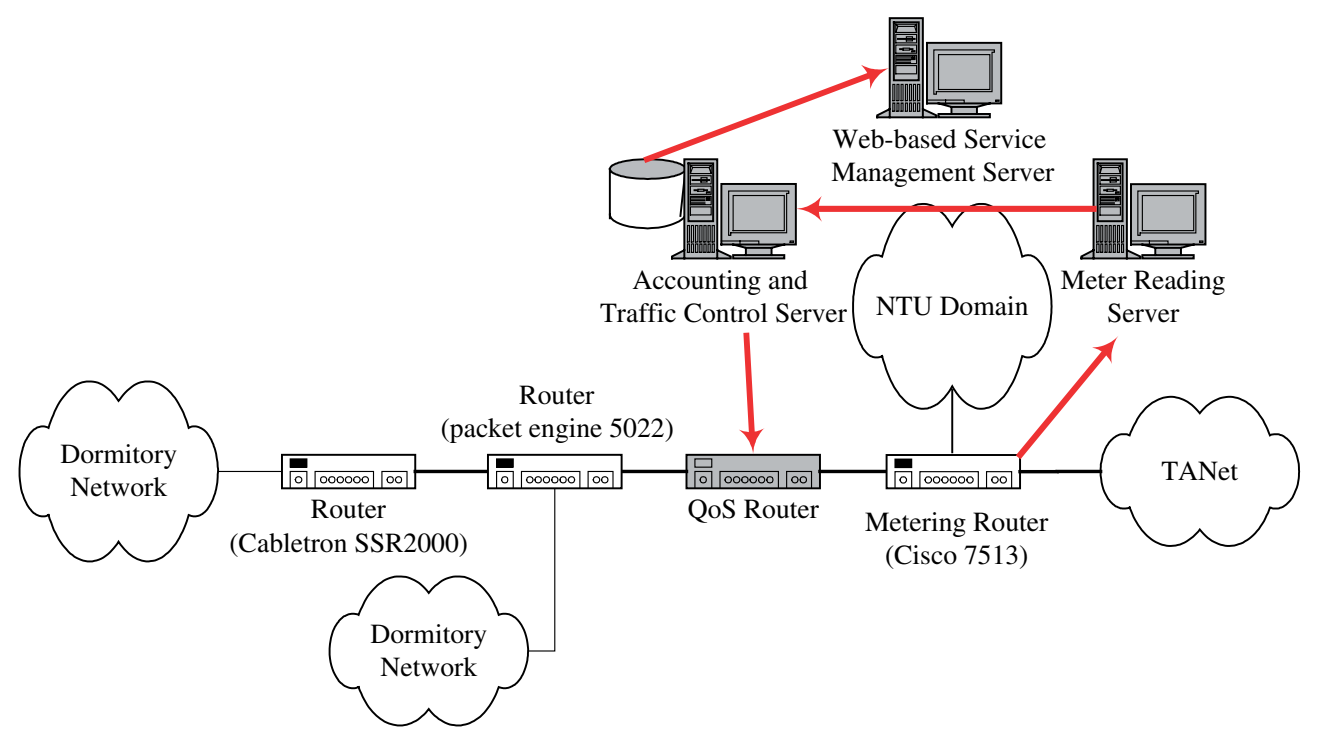

Fig. 6. Implementation of system architecture.

we exploit the existing hardware and software of the legacy production network as much as possible. There are five major component devices in this network management and control implementation [13]: (1) a quality of service (QoS) router, (2) a metering router, (3) a meter reading server, (4) an accounting and traffic control server, and (5) a Web-based service management server. All these devices but the metering router are new to the baseline network. Among the new devices, only the QoS router is inserted between two routers, Packet Engine 5022 and Cisco 7513; other new devices are add-ons to the production network and have no direct impact on packet transport.

\subsection{QoS router}

Sun et al. [14] have designed and developed a DiffServ QoS router, called DSQR. The router design was implemented for beta test in the NTU campus network. In view of its efficient performance and reliability, we adopted the router as our QoS router. We also conducted a one-week stress test of DSQR prior to its installation in our control system. It was tested at a peak rate of 100 Mbps regular-class traffic, $100 \mathrm{Mbps}$ custody-class traffic, and $50 \mathrm{Mbps}$ for each. During the period of one week, the router was very reliable and satisfactorily passed the stress test.

Fig. 7 depicts the functional blocks of the QoS router. The classifier and priority queuing scheduler of the router are specialized to the two-class service provisioning. The classifier checks both the source and destination IP addresses of a packet. If the packet is sent to the intranet (campus networks), it is always classified as belonging to the regular queue. Otherwise, it is necessary to check whether the quota of the source IP address is used up or not. If not, the packet is sent to the regular queue; otherwise, the packet goes to the custody queue. The scheduler sends out packets from the regular queue on a FIFO basis. Packets in the custody queue get transmitted on a FIFO basis only when the regular queue is empty.

\subsection{Metering router (Cisco router 7513)}

Netflow $^{\mathrm{TM}}$ run on this router meters the outbound traffic flows. The collected data items include source and destination IP addresses, source and destination port numbers, protocol type, the number of packets and the volume transmitted. These raw traffic statistics, collected by the Cisco router, are collected by the meter reading server every 10 minutes, and are used to calculate the 


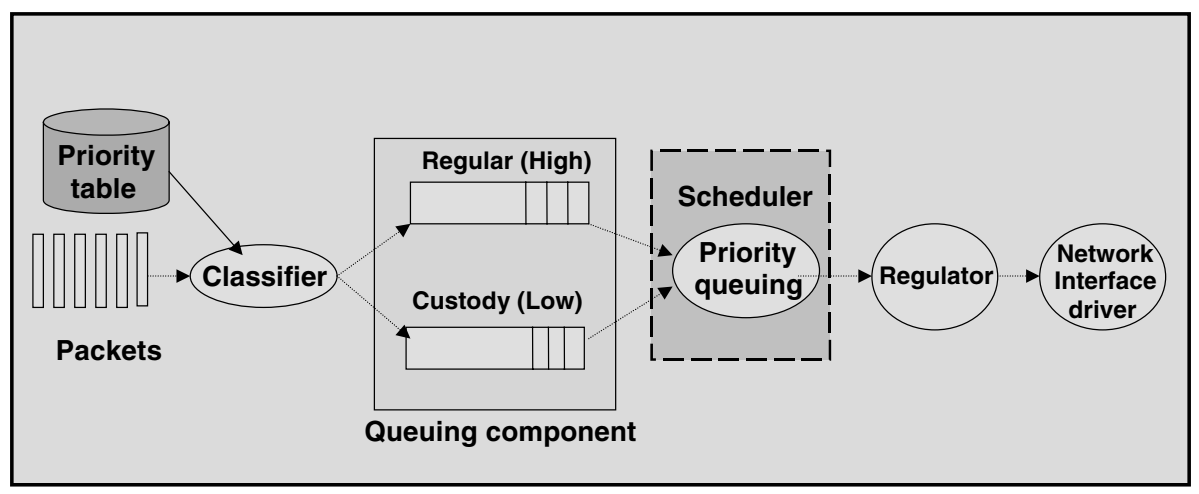

Fig. 7. Router architecture of DSQR.

cumulative volume usage per user for quota checking and users' behavior analysis.

\subsection{Meter reading server}

Fig. 8 shows the major components in the meter reading server. The data collector collects raw traffic statistics by FTP from the metering router based on Netflow. Then the information extractor written in Perl 5.0 ${ }^{\mathrm{TM}}$ extracts raw traffic statistics for traffic information items, such as the table of volume usage for each user per 10 minutes, the total application usage per 10 minutes and the volume dropped per 10 minutes. Finally, the information transmitter periodically exports the traffic information to the accounting/traffic control server via FTP.

\subsection{Accounting and traffic control server}

The server consists of two modules, one is for accounting and the other is for traffic control. As shown in Fig. 9, the accounting modules developed in Perl [15] calculate the available quota for the regular service in accordance with users' transmission volume for the Internet obtained by the meter reading server, and then update the accounting database. The accounting database stores the table of volume usage of each user per 10 minutes, the total application usage per 10 minutes, and the volume dropped per 10 minutes. Every 10 minutes, the accounting module then updates priorities of all IPs and sends the class change commands to the traffic control module.

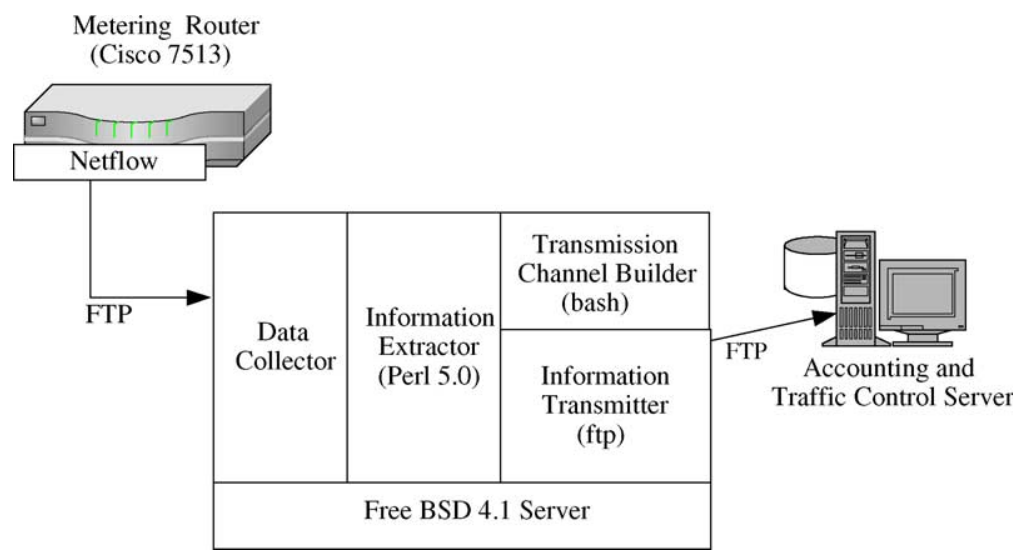

Fig. 8. Software architecture of meter reading server. 


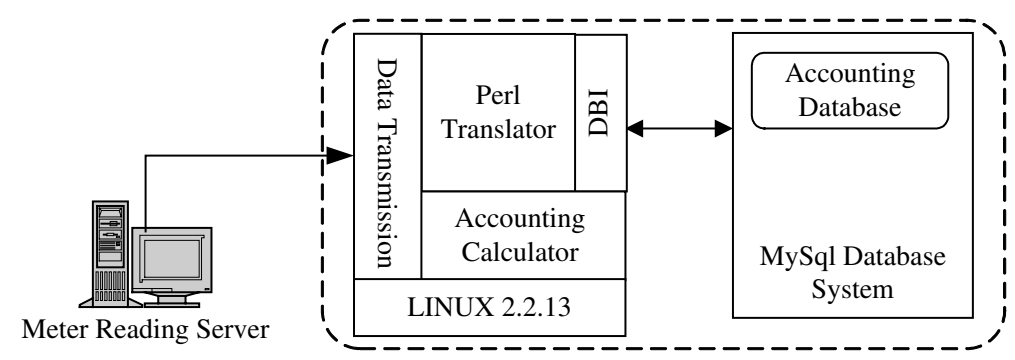

Fig. 9. Software architecture of accounting module.

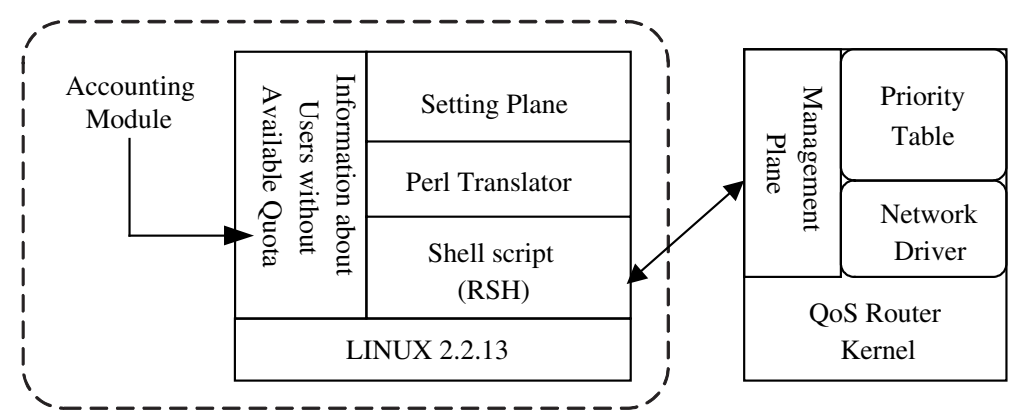

Fig. 10. Software architecture of traffic control module.

The traffic control module, shown in Fig. 10, is also implemented in Perl and generates the commands of shell scripts, which include IP addresses and their corresponding service classes. Then, it sends the commands to the DSQR router for priority control through the Remote SHell (RSH) communication protocol.

\subsection{Web-based service management server}

As shown in Fig. 11, a Web-based service management server constructed by using IIS $^{\text {TM }}$ (Internet Information Server) is set up at http:// ntunm.ntu.edu.tw. All its functionalities are developed in Active Server Page (ASP). On the Web site, users can check their available quota, their cumulative volume, and the current service class. The top heavy users and detailed traffic statistics are also displayed here. In addition, there are discussion and FAQ boards for users to feed back their comments for our policy or control parameters tuning.

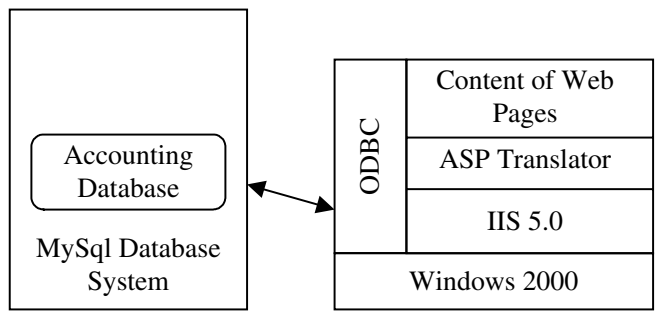

Fig. 11. Software of Web-based service management server.

In summary, the communication interactions among all devices are presented in a sequence diagram of Fig. 12.

\section{Experiment design and results}

An experimental study of the quota-based priority control system was then conducted over the dormitory networks. There were three objectives of the experiment study: 
1. to demonstrate the feasibility of our system implementation;

2. to assess the effectiveness of the quota-based priority control scheme;

3. to investigate user behavior under prioritized services with quota limitation and to validate user behavior and network performance models.

In the study, the main effectiveness measures include reduction of abuse, improvement of fairness, and alleviation of congestion. Both individual and group behavioral characteristics under the quota-based priority control were analyzed by examining experiment data such as volume distribution, daily profile of throughput and drop rate profile, and percentile by applications.

\subsection{Experiment design}

As described in Section 3, the quota value is the only design parameter in the control scheme proposed. It is therefore the control or independent variable in our experiment. The dependent variables of the experiment include (1) the volumes transmitted for each user every 10 minutes in the regular and the custody classes respectively, (2) volumes of packets dropped per 10 minutes for both the regular and the custody queues, and (3) total volume of each application per 10 minutes. The three can be used to calculate indices of interest according to the equations in Section 3: abuse index (Eq. (4)), unfairness index (Eq. (5)), throughput (Eq. (1)), drop rate (Eq. (2)), and volume of each user group by summing up the volume of individual users in the group.

We chose three quota levels for experimentation: infinite, $2 \mathrm{~GB}$ and $1 \mathrm{~GB}$. When the quota is infinite in the control scheme, there is essentially no control. A daily quota of 2 GB allows approximately $99 \%$ of users to have their daily demands satisfied via the regular service and $1 \mathrm{~GB}$ allows approximately $98 \%$. The quota is renewed daily at 6 a.m., when the outbound traffic of dormitory networks is the least. We experimented with infinite quota for 2 weeks, and 2 GB and 1 GB quota cases for 1 week each.
To convey the purposes and ideas of our experiment to users and to get their support, we gave briefings to dormitory and campus network managers and got their and users' feedbacks during implementation of the quota-based priority control system and the design of experiment. Such communications take place during the semester prior to our experiment. With the network managers convinced and willing to help, we then announce our experimental plan to all dormitory users through posting the announcement on the Bulletin Board System (BBS) and distribution of posters and flyers. A public hearing was also held before the experiment. We constructed a Webbased service management server to let users check the status of their accounts, experiment schedule and FAQs. This also facilitates direct communications among users and the research team for quick response to users' comments. Users then have a two-month trial period to familiarize themselves with the experiment system and to feed back their comments for system tuning. The installation of the QoS router requires less than one hour of service interruption.

Our hypotheses about this experiment are as follows:

(H1) The abuse would be reduced because the quota prevents HUs from abusive usage of the regular service. There would be two results of the abuse-reduction effect: First, the total volume of an abusive user transmitted via both regular and custody service classes would be reduced, which might lead to a lower average utilization of bandwidth. Second, some insignificant or not-so-urgent transmission demands would be shifted to off-peak hours, when there is no congestion and the quality of the custody class is comparable to that of the regular class, which might lower the difference between peak and off-peak hours.

(H2) Drop rate of the outbound traffic would be lowered and congestion alleviated because of less abusive and hence total traffic demands.

(H3) Fairness among users would be improved in that all users would enjoy better 


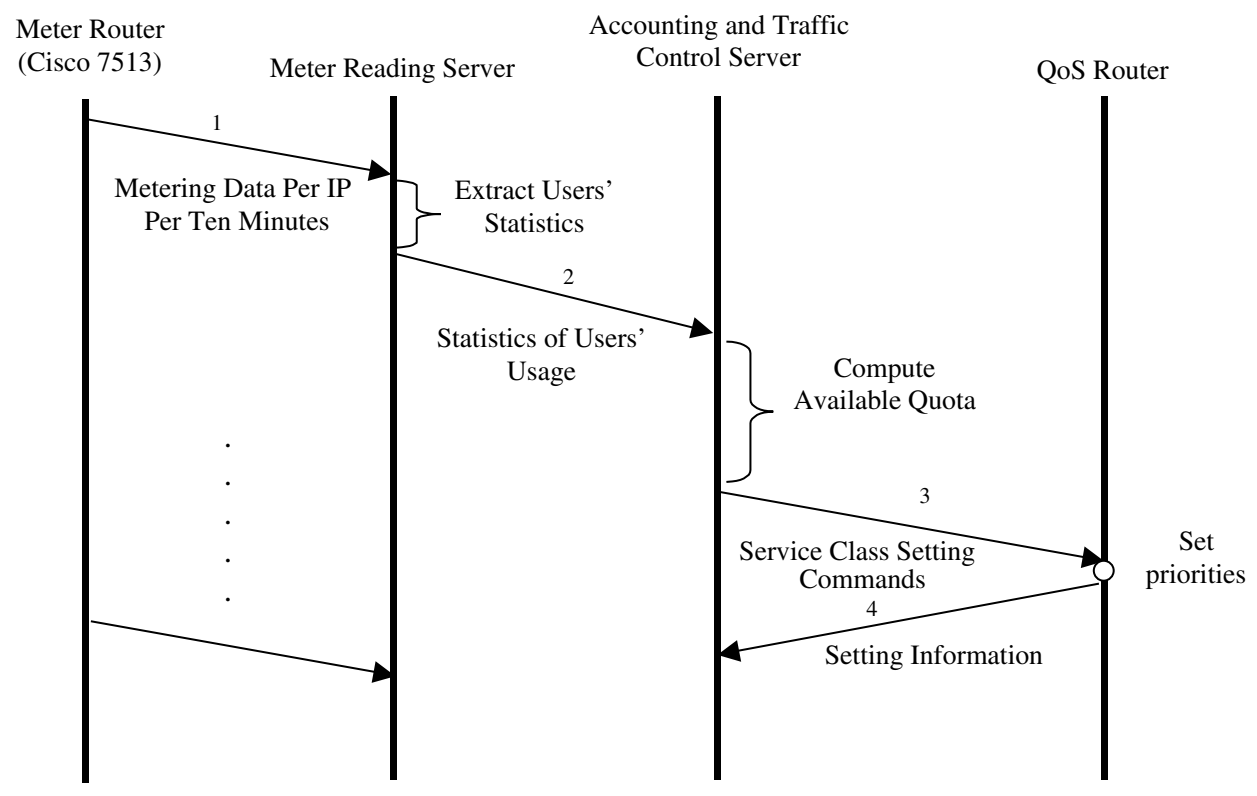

(a)

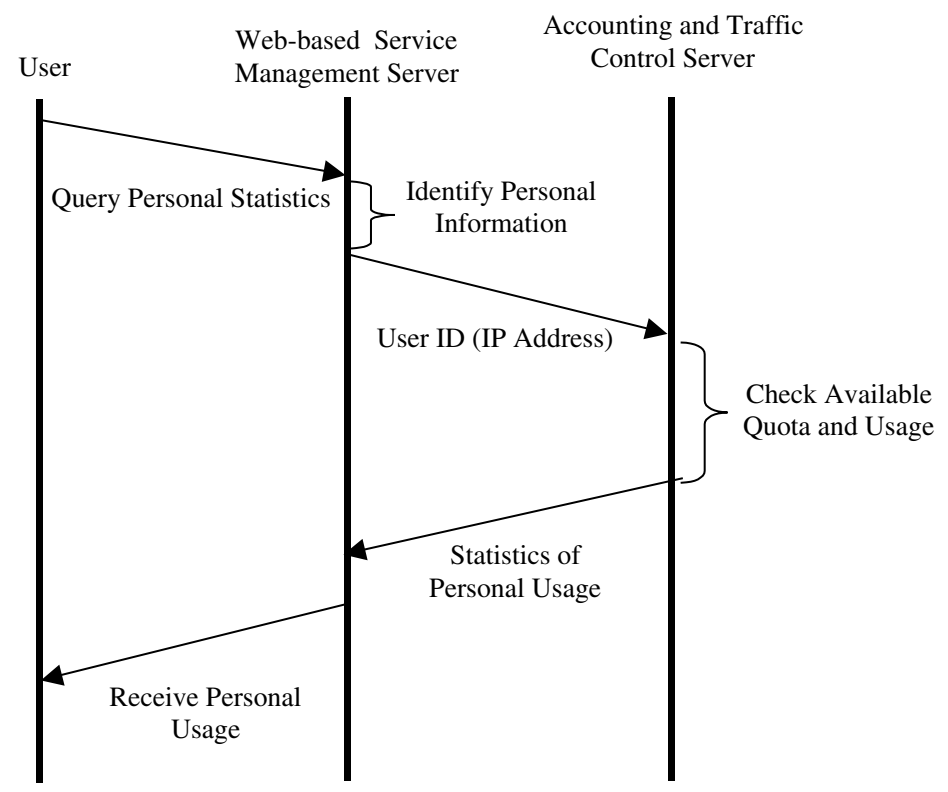

(b)

Fig. 12. Sequence diagram of all devices: (a) control traffic and (b) user traffic.

network performance, especially during the peak hours.
(H4) The usage of applications with a small volume per transmission would increase when 
Table 4

Comparisons of daily usage among user groups

\begin{tabular}{|c|c|c|c|}
\hline \multirow[t]{2}{*}{ Group } & \multicolumn{3}{|l|}{ Quota } \\
\hline & $\begin{array}{l}\text { Infinite } \\
\text { (Average bytes) }\end{array}$ & $\begin{array}{l}2 \text { GB } \\
\text { (Average bytes) }\end{array}$ & $\begin{array}{l}1 \mathrm{~GB} \\
\text { (Average bytes) }\end{array}$ \\
\hline 2GHU (45 users; >2 GB) & $3,846,103,572$ & $\begin{array}{l}1,326,036,309 \\
(-65.5 \%)^{\mathrm{a}}\end{array}$ & $\begin{array}{l}748,192,250 \\
(-80.6 \%)\end{array}$ \\
\hline 1GHU (62 users; $2 \mathrm{~GB} \sim 1 \mathrm{~GB}$ ) & $1,429,553,853$ & $\begin{array}{l}1,133,276,307 \\
(-20.8 \%)\end{array}$ & $\begin{array}{l}751,079,797 \\
(-47.5 \%)\end{array}$ \\
\hline 100MHU (329 users; $1 \mathrm{~GB} \sim 100 \mathrm{MB}$ ) & $353,783,149$ & $\begin{array}{l}357,655,232 \\
(+1.1 \%)\end{array}$ & $\begin{array}{l}332,494,357 \\
(+6.0 \%)\end{array}$ \\
\hline NU (3043 users; $100 \mathrm{MB} \sim 3162 \mathrm{~B}$ ) & $5,586,235$ & $\begin{array}{l}23,126,272 \\
(+314.0 \%)\end{array}$ & $\begin{array}{l}23,802,242 \\
(+326.1 \%)\end{array}$ \\
\hline LU (1876 users; <3162 B) & 170 & $\begin{array}{l}4,459,953 \\
(+2623530 \%)\end{array}$ & $\begin{array}{l}9,001,687 \\
(+5295290 \%)\end{array}$ \\
\hline Abuse index; Top 2\% users (GB) & 261.71 & $\begin{array}{l}129.92 \\
(-50.4 \%)\end{array}$ & $\begin{array}{l}80.22 \\
(-69.3 \%)\end{array}$ \\
\hline Unfairness index & 6.08 & $\begin{array}{l}3.92 \\
(-35.5 \%)\end{array}$ & $\begin{array}{l}3.45 \\
(-43.3 \%)\end{array}$ \\
\hline
\end{tabular}

${ }^{a}$ Percent of change compared to the value under infinite quota.

network performance is improved while the major applications used by HUs such as non-well-known applications, FTP and HTTP, would be reduced.

(H5) The lower the quota volume is, the more significant the effects described in $(\mathrm{H} 1)-(\mathrm{H} 4)$ would be.

Quantitatively, our model described in Section 3 predicts a $28.2 \%$ reduction of average demands, a $42.2 \%$ reduction of unfairness, and a $62.32 \%$ reduction of abuse when the quota value is set to $1 \mathrm{~GB}$.

\subsection{Reduction of abusive Internet access}

Experiment results listed in Table 4 show a $38.2 \%$ reduction of the abuse index value under 2 GB quota and $57.9 \%$ reduction under 1 GB quota. These results are close to the numbers (33.4 and $63.3 \%$ ) projected by our numerical experiment (Table 3). Detailed analyses by user groups show similar observations in the $2 \mathrm{GHU}$ and $1 \mathrm{GHU}$ groups. For example, under the $1 \mathrm{~GB}$ quota, there are $80.6 \%$ and $47.5 \%$ reductions in daily volume usage of $2 \mathrm{GHUs}$ and $1 \mathrm{GHUs}$, respectively. Fig. 13 gives an insight of 2GHUs' usage profile over a day, where a significant usage reduction occurs during 6 p.m. to 6 a.m. It indicates that the $2 \mathrm{GHU}$ users used up their $1 \mathrm{~GB}$ quota within 12 hours of their daily quota replenishment, which took place at 6 a.m., and could only access the custody class of service afterwards. Such behavior implies that the abusive usage is time insensitive, i.e., it takes place around the clock. As the 2GHUs exhaust their daily quota by the time the peak hours starts, they can only use the low priority custody service

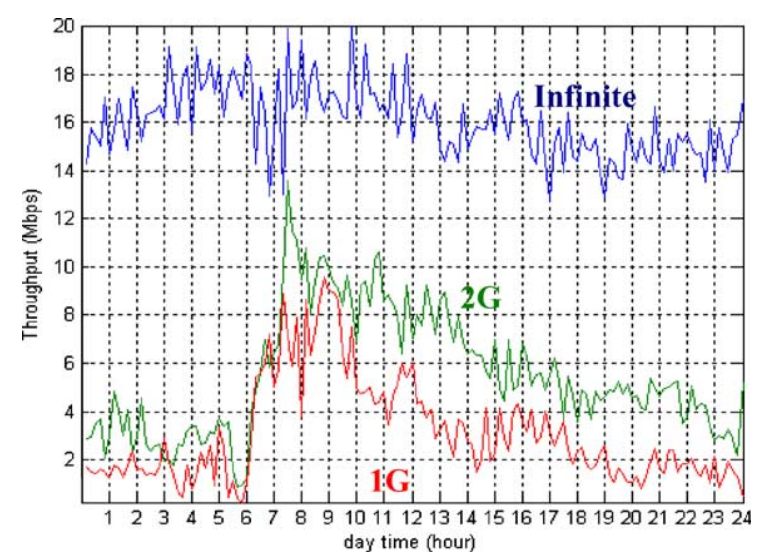

Fig. 13. Throughputs of the $2 \mathrm{GHU}$ group under various quota levels. 

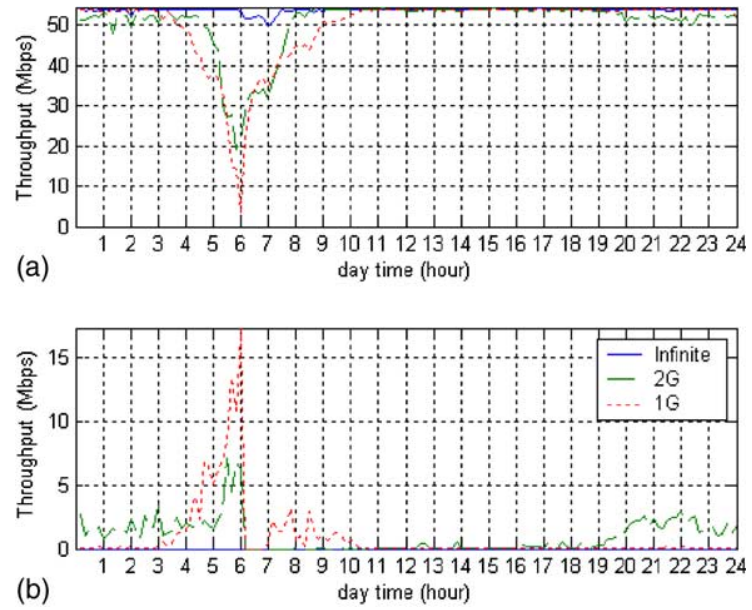

Fig. 14. Throughputs of two service classes under various quota levels: (a) regular class and (b) custody class.

during the peak hours and their usage is largely reduced. Fig. 14 shows a high throughput of custody class in the early morning hours (3 a.m.6 a.m.), indicating the demands of the top $2 \%$ users can still be satisfied by using network service at the time of lower capacity utilization. Network capacity is not wasted during off-peak hours either.

These results basically match our hypotheses (H1) and (H5). However, the usage increase in NU and LU groups during peak hours was unexpected. The reasons and implications of such a phenomenon will be discussed after examining the improvements of network performance under our control scheme.

\subsection{Network performance improvements}

As predicted by (H.1), (H.2) and (H.5), experimental results in Table 5 show that the daily average volume or throughput of Internet access is reduced by up to $29.8 \%$ and $21.4 \%$ at the $1 \mathrm{~GB}$ and 2 GB quota levels. Figs. 15-17 present the average daily performance profiles of Internet access in terms of the total throughput, throughput of regular service, and drop rate. The average is taken over the 7 days experiment for quota levels of 2 $\mathrm{GB}$ and $1 \mathrm{~GB}$, and the last 7 days for the infinite quota case.
Table 5

Control effect for drop rate and Internet access

\begin{tabular}{ccll}
\hline Internet access & Quota & & \\
\cline { 2 - 4 } & Infinite & $2 \mathrm{~GB}$ & $1 \mathrm{~GB}$ \\
\hline Drop rate & 5.95 & 3.98 & 3.04 \\
(Mbps) & & $(-33.1 \%)^{\mathrm{a}}$ & $(-48.9 \%)$ \\
Throughput & 32.89 & 25.85 & 23.09 \\
$\quad$ (Mbps) & & $(-21.4 \%)$ & $(-29.8 \%)$ \\
Mean volume & 73.78 & 60.94 & 52.09 \\
$\quad$ (MB) & & $(-21.4 \%)$ & $(-29.8 \%)$ \\
Standard devia- & 449.03 & 239.14 & 179.53 \\
tion (MB) & & $(-46.8 \%)$ & $(-59.9 \%)$ \\
\hline
\end{tabular}

${ }^{a}$ Percent of change compared to the value under infinite quota.

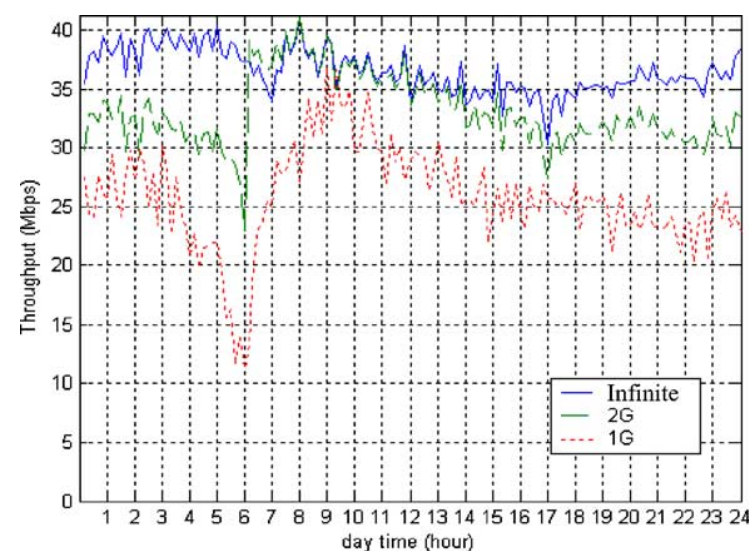

Fig. 15. The Internet access throughputs over a day under various quota levels.

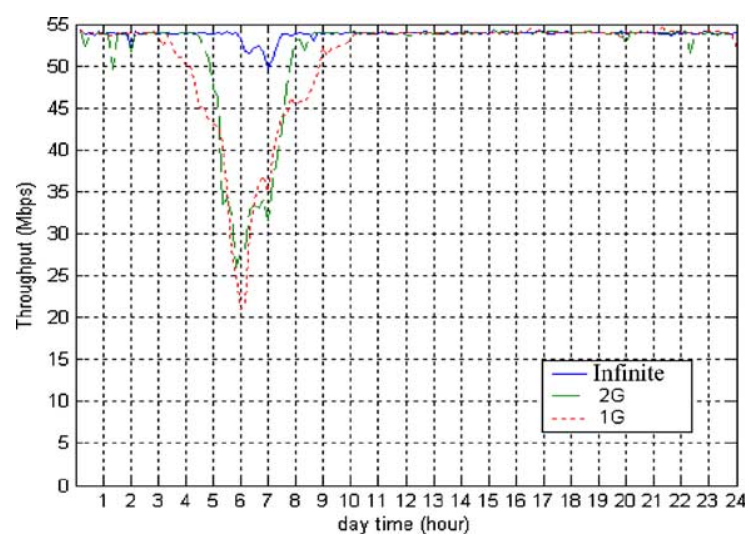

Fig. 16. Total throughputs under various quota levels. 


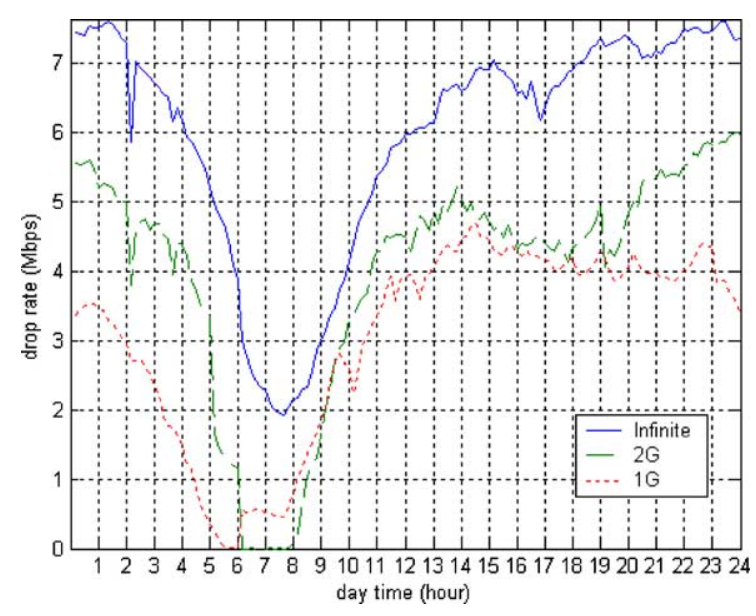

Fig. 17. Drop rates of regular class service under various quota levels.

Significant performance improvements take place during the peak hours; the total throughput and the drop rate decrease by $1.24 \%$ and $57.23 \%$ respectively during the period. The reduction of Internet access does encourage the usage increase of intranet access as shown in Fig. 18. But, the total traffic is still reduced and congestion alleviated. The throughput rate of the regular class drops by a maximum of $68.1 \%$ around 6 a.m. at

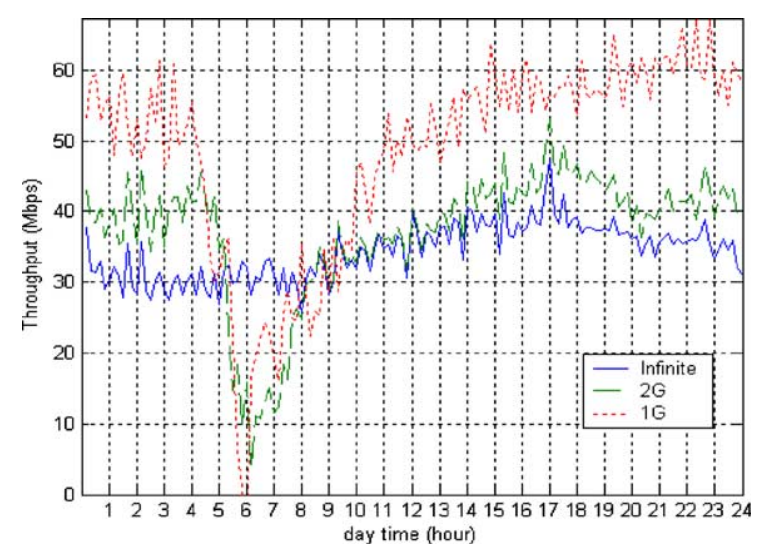

Fig. 18. Intranet traffic profiles under various quota levels.

the $1 \mathrm{~GB}$ quota level as shown by the ditch in Fig. 15. Recall that 6 a.m. is the quota replenishment time each day. The left (decreasing) half of the ditch, i.e., the drop before 6 a.m., is because the HUs run out of quota and the right (increasing) half of the ditch is because of quota replenishment.

\subsection{Fairness improvement}

When the quota scheme is applied, the fairness index is improved by $35.5 \%$ at the 2 GB level and

Table 6

Peak hour usage among user groups under various quota levels

\begin{tabular}{|c|c|c|c|}
\hline \multirow[t]{2}{*}{ Group } & \multicolumn{3}{|l|}{ Quota } \\
\hline & $\begin{array}{l}\text { Infinite } \\
\text { (Average bytes) }\end{array}$ & $\begin{array}{l}2 \text { GB } \\
\text { (Average bytes) }\end{array}$ & $\begin{array}{l}1 \mathrm{~GB} \\
\text { (Average bytes) }\end{array}$ \\
\hline 2GHU (45 users; >2 GB) & $149,185,329$ & $\begin{array}{l}29,866,454 \\
(-80.2 \%)\end{array}$ & $\begin{array}{l}17,004,173 \\
(-488.7 \%)\end{array}$ \\
\hline 1GHU (62 users; $2 \mathrm{~GB} \sim 1 \mathrm{~GB}$ ) & $78,103,520$ & $\begin{array}{l}56,390,983 \\
(-28.1 \%)\end{array}$ & $\begin{array}{l}24,530,722 \\
(-469.0 \%)\end{array}$ \\
\hline 100MHU (329 users; $1 \mathrm{~GB} \sim 100 \mathrm{MB}$ ) & $48,572,395$ & $\begin{array}{l}53,171,268 \\
(+8.9 \%)\end{array}$ & $\begin{array}{l}43,410,386 \\
(-11.4 \%)\end{array}$ \\
\hline NU (3043 users; $100 \mathrm{MB} 3162 \mathrm{~B}$ ) & 998,167 & $\begin{array}{l}4,556,856 \\
(+356.5 \%)\end{array}$ & $\begin{array}{l}4,754,360 \\
(+376.3 \%)\end{array}$ \\
\hline LU (1876 users; <3162 B) & 29 & $\begin{array}{l}823,564 \\
(+2839875.9 \%)\end{array}$ & $\begin{array}{l}1,736,643 \\
(+5988424.1 \%)\end{array}$ \\
\hline Abuse index; Top 2\% users (GB) & 32.96 & $\begin{array}{l}12.51 \\
(-62.0 \%)\end{array}$ & $\begin{array}{l}6.02 \\
(-81.7 \%)\end{array}$ \\
\hline Unfairness index & 5.96 & $\begin{array}{l}3.54 \\
(-40.6 \%)\end{array}$ & $\begin{array}{l}3.11 \\
(-47.8 \%)\end{array}$ \\
\hline
\end{tabular}




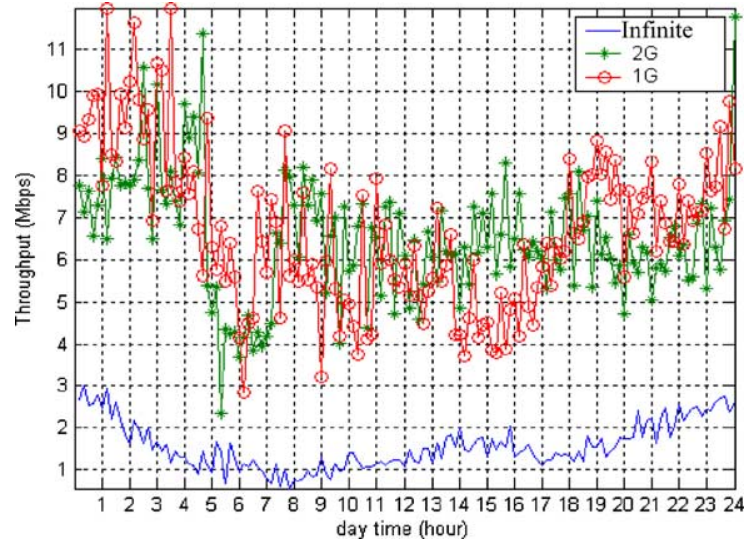

Fig. 19. Throughputs of NUs under various quota levels.

$43.3 \%$ at the $1 \mathrm{~GB}$ level, which are close to those projected $(32.7 \%$ and $42.2 \%)$ by our model in Table 3. Such improvements are due to usage reduction of 2 GHUs and 1 GHUs and usage increase of the NUs and LUs. For example, when the quota is $1 \mathrm{~GB}$, total daily volume of the top $2 \%$ users decreases by $57.88 \%$ while that of the majority users (NUs and LUs, 91.87\% of users)

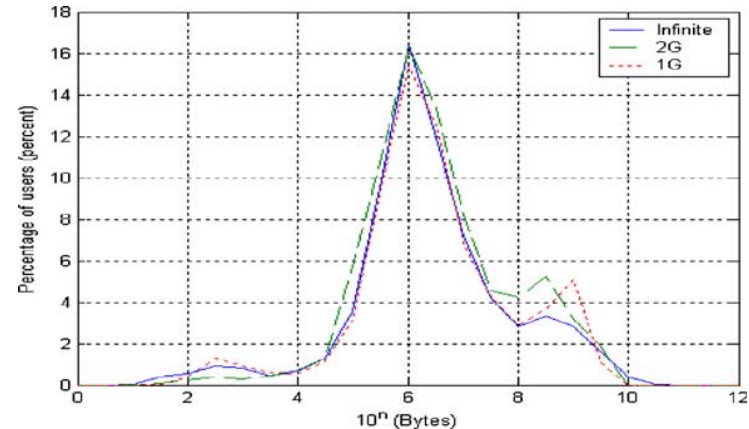

Fig. 20. Internet usage distribution under various quota levels.

increases by $487.2 \%$ (Table 4). Associating the fairness improvements with the reductions in throughput/utilization and drop rates, we deduce that the abusive usage is restricted and network performance is improved by our control scheme. As a result, normal usage is encouraged.

The fairness improvements are especially significant during the peak hours. On the one hand, Tables 4 and 6 show, at the 1 GB quota level, a $425.3 \%$ increase in daily volume and a $503.4 \%$ increase in peak-hour volume transmitted by the

Table 7

Comparisons of daily usage of all groups

\begin{tabular}{|c|c|c|c|}
\hline \multirow[t]{2}{*}{ Application } & \multicolumn{3}{|l|}{ Quota } \\
\hline & $\begin{array}{l}\text { Infinite } \\
\text { (total }=36.7475 \mathrm{Mbps})\end{array}$ & $\begin{array}{l}2 \mathrm{~GB} \\
(\text { total }=33.1658 \mathrm{Mbps})\end{array}$ & $\begin{array}{l}1 \mathrm{~GB} \\
(\text { total }=27.7109 \mathrm{Mbps})\end{array}$ \\
\hline OTHERS & $\begin{array}{l}35.0113 \\
(95.26 \%)\end{array}$ & $\begin{array}{l}31.6740 \\
(95.50 \%)\end{array}$ & $\begin{array}{l}26.3209 \\
(94.98 \%)\end{array}$ \\
\hline НTTP & $\begin{array}{l}1.1765 \\
(3.21 \%)\end{array}$ & $\begin{array}{l}0.9939 \\
(3.00 \%)\end{array}$ & $\begin{array}{l}0.7939 \\
(2.86 \%)\end{array}$ \\
\hline HTTPS & $\begin{array}{l}0.2253 \\
(0.61 \%)\end{array}$ & $\begin{array}{l}0.2422 \\
(0.73 \%)\end{array}$ & $\begin{array}{l}0.3734 \\
(1.34 \%)\end{array}$ \\
\hline Telnet/SSH & $\begin{array}{l}0.0805 \\
(0.22 \%)\end{array}$ & $\begin{array}{l}0.0723 \\
(0.22 \%)\end{array}$ & $\begin{array}{l}0.1006 \\
(0.36 \%)\end{array}$ \\
\hline FTP & $\begin{array}{l}0.0369 \\
(0.10 \%)\end{array}$ & $\begin{array}{l}0.0239 \\
(0.07 \%)\end{array}$ & $\begin{array}{l}0.0084 \\
(0.03 \%)\end{array}$ \\
\hline SMTP & $\begin{array}{l}0.0310 \\
(0.08 \%)\end{array}$ & $\begin{array}{l}0.0400 \\
(0.12 \%)\end{array}$ & $\begin{array}{l}0.0154 \\
(0.056 \%)\end{array}$ \\
\hline POP3 & $\begin{array}{l}0.0211 \\
(0.06 \%)\end{array}$ & $\begin{array}{l}0.0030 \\
(0.0090 \%)\end{array}$ & $\begin{array}{l}0.0030 \\
(0.011 \%)\end{array}$ \\
\hline NNTP & $\begin{array}{l}0.0008 \\
(0.0022 \%)\end{array}$ & $\begin{array}{l}0.0008 \\
(0.0024 \%)\end{array}$ & $\begin{array}{l}0.0010 \\
(0.0036 \%)\end{array}$ \\
\hline X-Windows & $\begin{array}{l}0 \\
(0 \%)\end{array}$ & $\begin{array}{l}0 \\
(0 \%)\end{array}$ & $\begin{array}{l}0 \\
(0 \%)\end{array}$ \\
\hline
\end{tabular}


majority of users, such as NUs (Fig. 19) and LUs. On the other hand, they show a $69.3 \%$ reduction in daily volume and $81.7 \%$ reduction in peak-hour volume transmitted by the top $2 \%$ heavy users. Detailed analyses of 2GHUs' volume transmitted over time confirm our hypothesis that they just constantly transmit and use up their daily quota by the peak hour starts. So the 2GHUs have a largest volume reduction among HUs in peak hours. The shifts in user distribution are given in Fig. 20, where there is a decrease in the numbers of 1 GHUs, 2GHUs and LUs and an increase in the numbers of NUs and 100MHUs. These experimental results basically match our hypotheses (H3) and (H5).

\subsection{Analysis by applications}

In Table 7, throughputs significantly decrease in applications such as OTHERS, HTTP and FTP, which are popular to HUs. The throughputs of HTTPS and TELNET, which are two of the major applications of LUs and NUs have significant increase. Such observations reconfirm that abusive usage is restricted and normal usage encouraged. However, the percentiles of Table 7 indicate that the traffic percentage of OTHERS stays above $95 \%$ and is insensitive to our quota-based priority control, which was not predicted by our hypothesis (H4). Other methods have to be designed to answer the challenge of (10).

\section{Conclusions}

In this paper, a quota-based priority control scheme has been designed to manage unfair and abusive Internet access encountered in the NTU dormitory networks. There are two classes of services in the scheme, where the regular class sets a quota on each user's traffic volume per quotacontrol period and the out-of-profile or possibly abusive traffic is directed to the lower priority (custody) class of service. The goal is to meet the basic traffic demands of the majority users while limiting abusive usage from heavy users. The quota-based priority control has been realized by adding to the NTU legacy networks a QoS router, a meter reading server, an accounting server and a Web-based service management server with minimal intrusion of user privacy and least disturbance to the existing service offering. Experimental results over the 5335 user population show that under the control, abusive Internet access by the top $2 \%$ heavy users is reduced by $57.9 \%$ and accordingly, the congestion is alleviated by a $48.9 \%$ reduction of average packet drop rate. There is also a $43.3 \%$ reduction in the coefficient of variation in users' daily usage. Furthermore, with the improvement in peak-hour congestion, the traffic volume generated by the majority of users increases by $503.4 \%$. The scheme clearly leads to better fairness among users. As the heavy users can still access the custody service class after running out of quota and get comparable service quality to the regular service class during the off-peak hours, network resources are still efficiently utilized in offpeak hours. All users have been pleased with this win-win control scheme. In addition, mathematical models of user behavior and network performance have also been constructed. The high consistency between the experimental results and the prediction by the models indicates good model validity.

\section{Acknowledgements}

We would first like to thank Pei-Chun Cheng and Pei-Wen Chen at NTU, and Yin-Chang Lin, Guan-Chuan Wang and Dr. Meng Chang Chen at the Institute of Informaton Science of Academia Sinica for providing us their implementation of the most important component - the QoS router-in our experiments. Without their system design and code, and continuing technical support, this field-trial experiment would not have been possible.

The authors would also like to thank Yu-Qun Pan, Guang-Wei Li, Yu-Mei Shao, Yin-Ren Chien, Zhi-Xiu Lin and Yu-Quan Li for very valuable discussions and technical support at various stages of this work. 


\section{Appendix A}

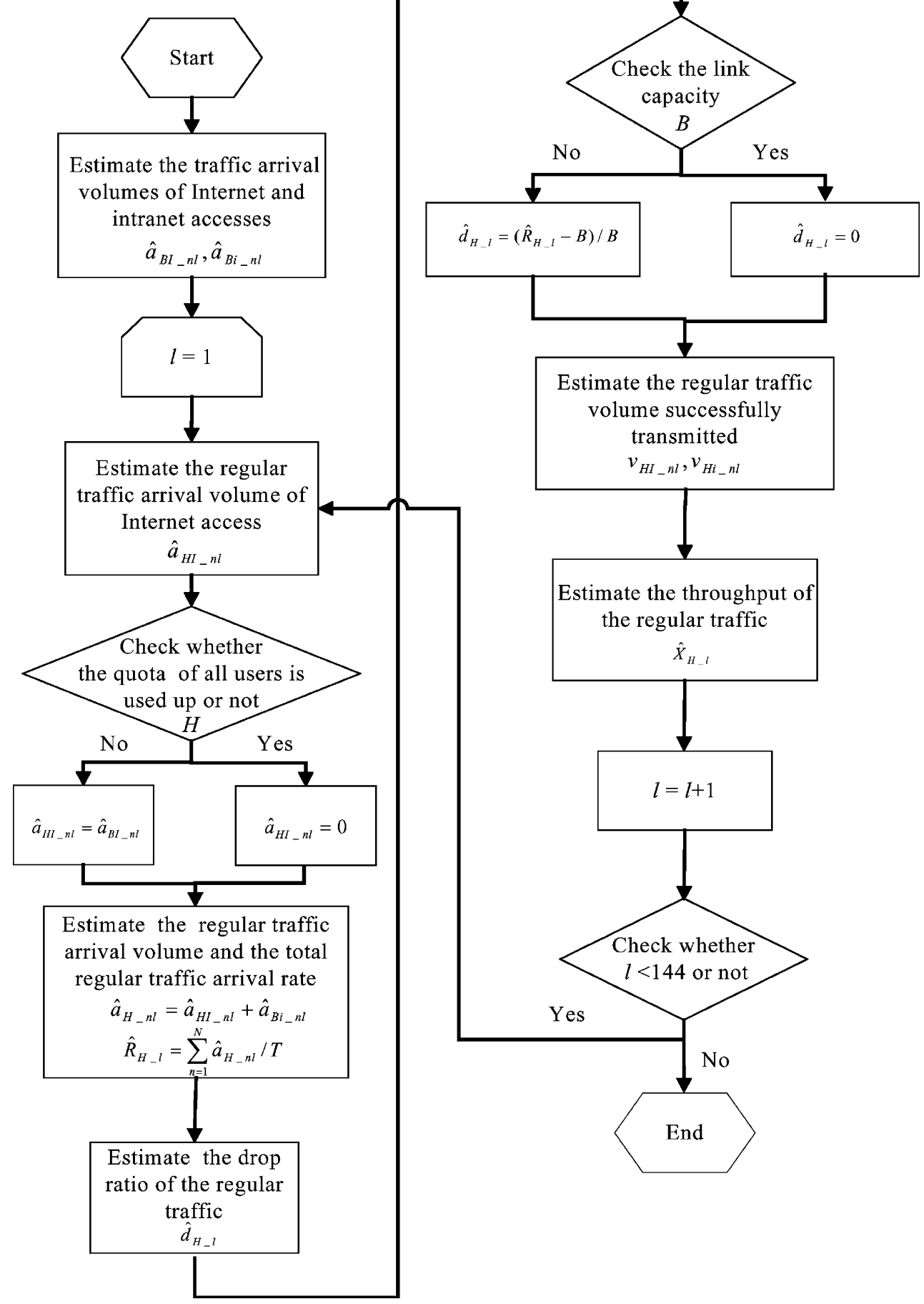




\section{References}

[1] F. Kelly, A. Maulloo, D. Tan, Rate control for communication networks: shadow price proportional fairness and stability, Journal of Operations Research Society 49 (1998) 237-252.

[2] Y. Le Boudec, Rate adaptation, congestion control and fairness: a tutorial. Available from <http://www.statslab. cam.ac.uk/ frank/pf/>.

[3] F. Kelly, Charging and rate control for elastic traffic, European Transactions on Telecommunications 8 (1997) 33-37.

[4] L. Massoulie, J. Roberts, Bandwidth sharing: objectives and algorithms, INFOCOM 99, 1999.

[5] Y. Zheng, Z. Feng, A new fairness criterion and its realization by using a new scheduling algorithm in the Internet, in: Proceedings of Sixth IEEE Symposium on Computers and Communications, 2001, pp. 444-449.

[6] R. Edell, P. Varaiya, Providing Internet access: what we learn from INDEX, IEEE Network 13 (5) (1999) 18-25.

[7] S. Saroiu, K.P. Gummadi, R.J. Dunn, S.D. Gribble, H.M. Levy, An analysis of Internet content delivery systems, in: Proceedings of the 5th Symposium on Operating Systems Design and Implementation (OSDI), 2002.

[8] P. Xu, Q. Wu, X. Dai, Token-passing linear dynamic quota function and its quality, in: International Conference on Communication Technology Proceedings (ICCT), 1996.

[9] S.-T. Cheng, C.-M. Chen, I.-R. Chen, Dynamic quotabased admission control with sub-rating in multimedia servers, Multimedia Systems 8 (2) (2000) 83-91.

[10] S. Blake, D. Black, M. Carlson, E. Davies, Z. Wang, W. Weiss, An architecture for differentiated services, IETF RFC 2475, 1998. Available from <http://www.ietf.org/rfc/ rfc2475.txt>.

[11] A. Giovanardi, G. Mazzini, Analysis and implementation of a transparent priority mechanism for LAN Internet access, GLOBECOM '99, 1999.

[12] http://www.cisco.com/warp/public/732/Tech/netflow.

[13] Y.-T. Chou, Design and implementation of virtual Internet pricing system in campus network, Master Thesis, Department of Electrical Engineering, National Taiwan University, 2001.

[14] Y.S. Sun, J.-F. Lee, Policy-based QoS management in NBENdifferentiated services provisioning, TANET'2000, 2000.

[15] http://www.perl.com.

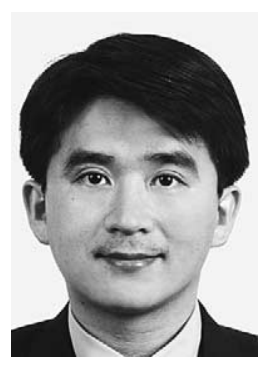

Tsung-Ching Lin received the B.S. degree in Mechanical Engineering from Chung-Yuan Christian University, Taiwan, Republic of China, in 1993 and the M.S. degree in Mechanical Engineering from National ChiaoTung University in 1995, respectively. He is currently a Ph.D. student in the Department of Electrical Engineering at National Taiwan University. His current research interests include Internet traffic control, network management and Internet pricing.

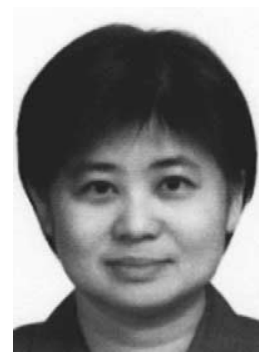

Yeali S. Sun was born in Taipei, Taiwan. She received her B.S. degree in Computer Science from National Taiwan University in 1982, and the M.S. and Ph.D. degrees, both in Computer Science, from the University of California, Los Angeles (UCLA) in 1984 and 1988, respectively. From 1988 to 1993, she was with Bell Communications Research Inc. (Bellcore; now Telcordia), where she was involved in the area of planning and architecture design of information networking, broadband networks, and network and system management. In August 1993, she jointed National Taiwan University and is currently a professor of the Department of Information Management. She was the director of the Information Networking Group in the Computer Center of National Taiwan University, responsible for managing the university campus network and TANet Northern Regional POP (Point of Presence) and leading an advanced networking research team prototyping and conducting a series of field trials and experiments on policy-based QoS delivery services, bandwidth management, and Internet pricing for congestion control. In 1996-2002, she served in the TANet Technical Committee, Steering Committee of the National Broadband Experimental Network (NBEN) and Internet2, and IP Committee of TWNIC. Her research interests are in the area of network architecture and protocols, and implementation of high-performance content switch, in particular regarding quality of service (QoS), fast content classification, content delivery, Internet pricing and network management.

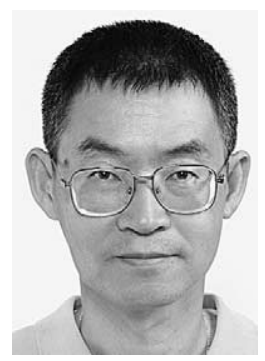

Shi-Chung Chang (S'83-M'87) received his B.S.E.E. degree from National Taiwan University, Taiwan, Republic of China, in 1979, and his M.S. and $\mathrm{Ph} . \mathrm{D}$. degrees in electrical and systems engineering from the University of Connecticut, Storrs, in 1983 and 1986 respectively.

From 1979 to 1981 he served as an Ensign in the Chinese Navy, Taiwan. He worked as a technical intern at the Pacific Gas and Electric Co., San Francisco, in the summer of 1985. During 1987, he was a member of the Technical Staff, decision systems section, ALPHATECH, Inc., Burlington, MA. He has been with the Electrical Engineering Department of National Taiwan University since 1988 and was promoted to Professor in 1994. During 2001-2002, he served as the Dean of Student Affairs and a Professor of Electrical Engineering, National Chi Nan University, Pu-Li, Taiwan. He is now jointly appointed by the Graduate Institute of Industrial Engineering and the Graduate Institute of Communication Engineering, National Taiwan University, as well. His research interests include optimization theory and algorithms, operation scheduling and control of large-scale systems, high speed networks, Internet economics and distributed decision making. He has been a principal investigator and consultant to many industry and government funded projects in the above areas, and has published more than 120 technical papers. He received, in 1996, the award of outstanding achievements in University-Industry Collaboration by Ministry of Education for his pioneering and successful research collaborations with Taiwan semiconductor industry on production scheduling and control. He is a member of Eta Kappa $\mathrm{Nu}$ and Phi Kappa Phi. 


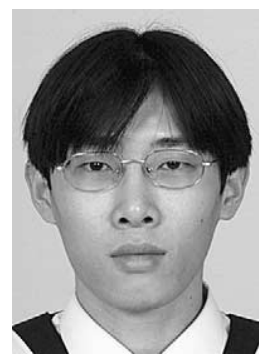

Shao-I Chu received the B.S. degree in Industrial Engineering from National Tsing Hua University in 1998 and the M.S. degree in Electrical Engineering from National Taiwan University in 2000 , respectively. $\mathrm{He}$ is currently a Ph.D. student in the Department of Electrical Engineering at National Taiwan University. His current research interests include statistical communication theory, network management and Internet pricing.

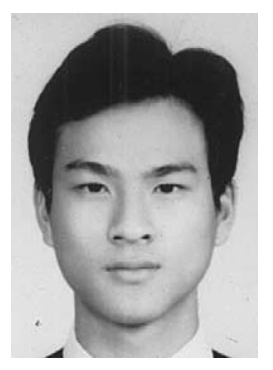

Yi-Ting Chou received the B.S. degree in Electrical Engineering from TamKang University in 1985 and the M.S. degree in Electrical Engineering from National Taiwan University in 2001, respectively. He is currently a software engineer in BENQ Corporation.

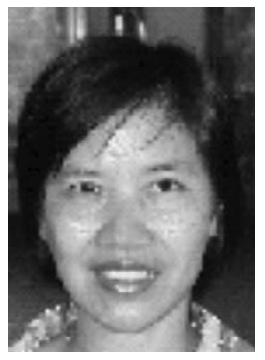

Mei-Wen Li received her M.S. degree in Computer Science from California Polytechnic State University, San Luis Obispo (CAL POLY) in 1992. She is a network administrator of Information Networking Group in the Computer Center of National Taiwan University, responsible for managing the university campus network and security. 